\title{
STARK BROADENING OF UV NICKEL LINES
}

\author{
ROGER D. BENGTSON \\ Dept. of Physics, University of Texas at Austin, Austin, Tex., U.S.A. \\ and \\ MYRON H. MILLER and R. A. ROIG \\ Institute for Fluid Dynamics and Applied Mathematics, University of Maryland, \\ College Park, Md., U.S.A.
}

Widths of Stark broadened Nir Lines in the spectral range of $3000 \AA-4000 \AA$ have been measured using a gas driven shock tube as a spectroscopic source. Electron densities were determined using measured pressure and temperature data. Within the density range $6 \times 10^{16} \mathrm{~cm}^{-3}$ to $12 \times 10^{16} \mathrm{~cm}^{-3}$ the observed widths were linear functions of the electron density. There was no apparent correlation of observed widths with neutral density. Van der Walls broadening was presumed to be negligible because we observed narrow lines which should have had the same Van der Walls widths as computed with a classical theory as some of the wide lines we observed. In this way we were able to attribute almost all of the observed broadening to the Stark effect. The experimental results were compared with results of calculations of Stark broadening parameters using the program developed by Cooper and Oertel. The measured widths were about a factor three larger than the theoretical predictions. A part of this discrepancy is probably due to the fact that only LS allowed interactions were considered in the broadening calculations and possible incomplete classifications.

\section{DISCUSSION}

H. Nussbaumer: You mention that refined calculations would bring good agreement with experiment. Unless you have definite indications I think it rather dangerous to make such a statement.

R.D. Bengtson: The point is well made. The statement I wish to make is that taking account of non LS allowed transitions should increase the calculated Stark widths.

D.D. Burgess: Do you have any check on optical depth other than the Lorentz shape of your profiles already mentioned?

R.D. Bengtson: Yes. We use a line reversal method of measuring temperature, which gives a direct measurement of the optical depth at one wavelength. Then from the photographically recorded spectrum we know the optical depth at all wavelengths. 